\title{
Disk-Jet Connection in Active Super-massive Black Holes in the Standard Accretion Disk Regime
}

\author{
Yoshiyuki Inoue* \\ Interdisciplinary Theoretical \& Mathematical Science Program (iTHEMS), RIKEN, 2-1 \\ Hirosawa, Saitama 351-0198, Japan \\ Kavli Institute for the Physics and Mathematics of the Universe (WPI), UTIAS, The University of \\ Tokyo, Kashiwa, Chiba 277-8583, Japan \\ E-mail: voshiyuki.inouedriken.jp
}

We study the disk-jet connection in supermassive black holes by investigating the properties of their optical and radio emissions utilizing the SDSS-DR7 and the NVSS catalogs. Our sample contains 7017 radio-loud quasars with detection both at $1.4 \mathrm{GHz}$ and SDSS optical spectrum. We find that the jet powers correlate with the bolometric disk luminosities as $\log P_{\text {jet }}=(0.96 \pm 0.012) \log L_{\text {disk }}+(0.79 \pm 0.55)$. This suggests that the jet production efficiency of $\eta_{\text {jet }} \simeq 1.1_{-0.76}^{+2.6} \times 10^{-2}$ assuming the disk radiative efficiency of 0.1 implying low black hole spin parameters and/or low magnetic flux for radio-loud quasars.

Accretion Processes in Cosmic Sources - II - APCS2018

3-8 September 2018

Saint Petersburg, Russian Federation

\footnotetext{
* Speaker.
} 


\section{Introduction}

Relativistic jets launched by supermassive black holes (SMBHs), so-called as active galactic nuclei (AGNs), are known as the most energetic particle accelerators in the universe. Because of their gigantic power, those jets would affect the fate of galaxies, and also galaxy clusters [ए2]. However, the launching mechanism of a collimated relativistic jet from a black hole system is a long standing problem in astrophysics.

Theoretically, the Blandford-Znajek (BZ) mechanism [团] is believed as the plausible explanation for the jet launch. In the BZ mechanism, the jet power is extracted by the rotation of BHs with the support of the magnetic fields threading the central $\mathrm{BH}$. Although observational evidence for the jet production mechanisms in AGNs are not clear yet, for example, [53] argued that spin parameter would determine the AGN radio loudness distribution.

It is also possible to investigate the jet production mechanisms from observations by probing the relation between disk inflow and jet outflow. Recent systematic spectral analysis for luminous blazars by [[5] shows the jet power is slightly larger than the power of accreting plasma, although the results depend on the assumptions on the pair fraction and the minimum energy of electrons [R], 40]]. On the contrary to blazars, in radio quasars, the efficiency of jet energy extraction from rest energy of accreted masses, i.e. the jet production efficiency, is not well understood yet.

Here, after the discovery of quasars, their radio emission is found to be associated with a presence of luminous optical emission lines. A correlation between radio jet luminosities and disk emission line luminosities in radio galaxies is later found [2], [46, 44, [4], 6], [6], 6, [28, [2]]. The correlation of radio jet luminosities and various optical emissions has been studied in more detail as the mass of the nuclear BH became available [64]. The BH mass provide fundamental information for the jet study such as the Eddington luminosity and its ratio.

Using the $\mathrm{BH}$ mass information, $\mathrm{BH}$ systems have been found to have a fundamental plane among mass, disk luminosity, and jet power [ [58, [36, B]]. [36] established the fundamental plane in stellar mass and supermassive BHs using BH mass, jet core radio luminosity, and disk X-ray luminosity. With $\sim 150 \mathrm{BH}$ system samples, they found that the radio core luminosity is correlated with both the mass and the X-ray luminosity of the disk. However, AGN disk luminosity is known to be dominated not in X-ray but in optical [ए]]. Moreover, as recent studies suggest that the fundamental plane exist only for low accretion rate $\mathrm{BH}$ systems [ [35, , 4]]], a fundamental plane for objects accreting at high rates is not well established.

The Sloan Digital Sky Survey (SDSS) [6]] has facilitated studies of AGNs in optical with wide and deep field surveys [49]. SDSS has detected 166583 quasars in optical covering about a quarter of the sky [BQ]. For the radio data, using Very Large Array (VLA), the NRAO VLA Sky Survey (NVSS) has observed the entire sky north of $-40 \mathrm{deg}$ declination at $1.4 \mathrm{GHz}$ down to $\sim 2.5 \mathrm{mJy}$ [ $[8]$ ]. NVSS has detected $\gtrsim 1.8$ million sources. In this paper, we study the relation among the jet power $\left(P_{\text {jet }}\right)$, the bolometric disk luminosity $\left(L_{\text {disk }}\right)$, and the black hole mass $\left(M_{\mathrm{BH}}\right)$ using quasar samples detected both in SDSS and NVSS which will allow us to investigate the disk-jet connection in the largest ever radio quasar sample. 


\section{Sample}

The NVSS was carried out utilizing the VLA radio interferometric telescopes at a frequency of $1.4 \mathrm{GHz}$ [ [8]. The NVSS was conducted with the array D configuration which provides a spatial resolution of 45 arcsec. Although this resolution is not as good as that of the Faint Images of the Radio Sky at Twenty-cm (FIRST) [3] having $\sim 5 \operatorname{arcsec}$, the NVSS resolution secures to measure fluxes of extended sources more accurately [B] $]$.

The NVSS covers the entire sky north of -40 deg declination $\left(\sim 33,000 \mathrm{deg}^{2}\right)$ and contains over 1.8 million sources down to a limiting flux density of $\sim 2.5 \mathrm{mJy}$. The survey gives astrometric accuracy ranges from 1 arcsec for bright sources to $\sim 7 \operatorname{arcsec}$ for faint sources. We extract integrated flux densities from the NVSS catalog to evaluate the whole extended radio flux.

The SDSS is an optical imaging and spectroscopic survey [65] using a $2.5 \mathrm{~m}$ wide-filed telescope at the Apache Point Observatory [1[8]. The photometric survey contains five wavelength bands [14]]. The survey covers about a quarter of the sky. A subset of photometric sources are chosen for spectroscopic observation according to the spectral target selection algorithms of SDSS. The survey catalog now contains 166583 quasars [B]]. Here, [49] provided detailed spectral properties of the SDSS data release (DR) 7 quasar catalog [47] which contains 105783 quasars brighter than $M_{i}=-22.0$. In this paper, we utilize the SDSS DR7 quasar catalog provided by [4⿴囗十) as we are interested in the spectral properties of quasars.

We match the NVSS radio sources and the DR7 quasar catalog with a matching radius of 30 arcsec and select the closest sources between the catalogs. As we are targeting SDSS detected quasars, the dominant radio quasar population is expected to be Fanaroff-Riley Class II (FR-II) [ए]3] whose radio brightest component, so-called hot spot, locates within several hundreds kpc away from the nucleus [37] which corresponds to $\sim 30 \operatorname{arcsec}$ at $z \sim 1-3$. After the catalog matching, the resulting number of objects detected both in radio and optical is 8436 .

We are interested in the relation between the jet and the accretion disk, i.e. radio-loud objects. Radio emission from radio-quiet quasars $(R<10)$ is expected to be associated with shocks produced by quasar driven outflows rather than jets [66]. To select radio-loud quasars, we select sources having $R \geq 10$ for the main sample which includes 7017 quasars.

\section{Relation between Jet Outflow and Accretion Disk Inflow}

The jet power is known to be correlated with the radio luminosity [63, [8]]. The empirical relation gives the time-averaged jet power from the extended radio luminosity as [63]

$$
P_{\text {jet }}=9.5 \times 10^{46}\left(\frac{f}{10}\right)^{3 / 2}\left(\frac{L_{151 \mathrm{MHz}}}{10^{28} \mathrm{~W} \mathrm{~Hz}^{-1} \mathrm{sr}^{-1}}\right)^{6 / 7}\left[\mathrm{erg} \mathrm{s}^{-1}\right]
$$

where $f$ is a parameter accounting for systematic error in the model assumptions. [63] constrained as $1 \leq f \lesssim 20$ by using X-ray cavity measurements. In this paper, we set $f=10$ following the recent $\mathrm{X}$-ray cavity and hot spot studies [[5, [1]]. We note the power given in $[$.$] is the time-averaged value.$

Since our samples are quasars, $L_{\text {disk }}$ can be related to the mass accretion rate $\dot{M}_{\text {in }}$ onto the SMBH as $L_{\text {disk }}=\varepsilon \dot{M}_{\text {in }} c^{2}$, where $\varepsilon$ is the accretion disk radiative efficiency and $c$ is the speed of light. Fig. $\square$ shows the correlation between the bolometric disk luminosity $L_{\mathrm{disk}}$ and the jet power 


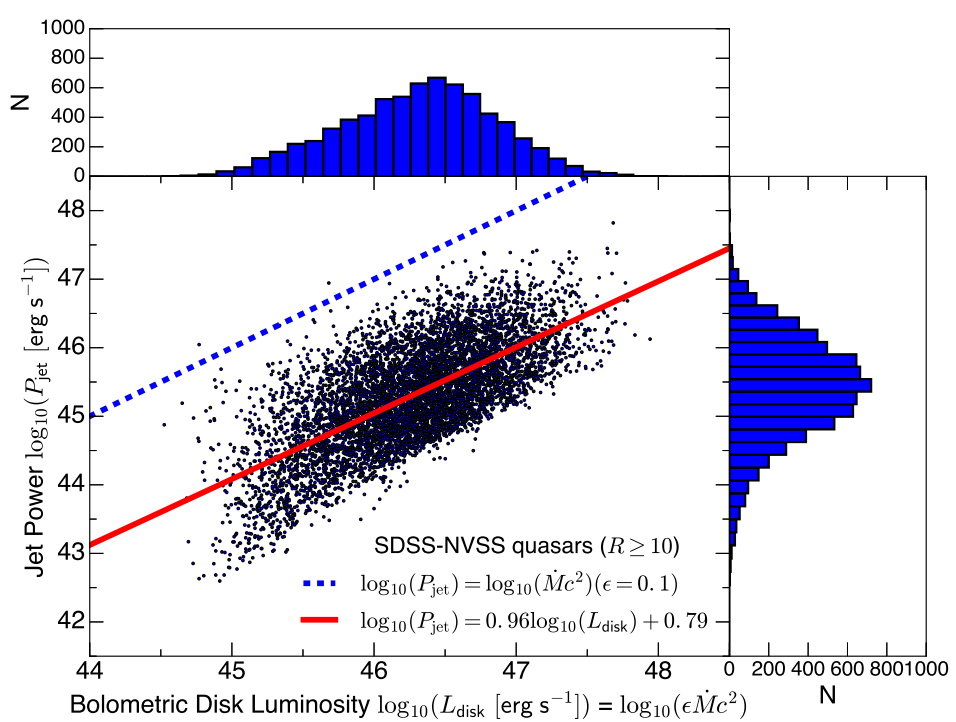

Figure 1: Bolometric disk luminosity vs. jet power of our SDSS-NVSS sample, where the top and right panels show the bolometric disk luminosity and jet power histograms. The solid line gives a linear fit to the data with a scatter of 0.74 . The dashed line shows the case with the jet production efficiency $\eta_{\text {jet }}=1$ assuming the disk efficiency $\varepsilon=0.1$.

$P_{\text {jet }}$ in the logarithmic space. The top and bottom panel shows the histogram of $\log \left(L_{\text {disk }}\right)$ and $\log \left(P_{\mathrm{jet}}\right)$, respectively. The linear regression line, shown by the solid line, is given as

$$
\log P_{\text {jet }}=(0.96 \pm 0.012) \log L_{\text {disk }}+(0.79 \pm 0.55)
$$

where errors show $1 \sigma$ uncertainties, with a scatter of 0.54 . We perform a partial correlation analysis to test the correlation between the $\log L_{\text {disk }}$ and $\log P_{\text {jet }}$ excluding the redshift dependence. The Spearman rank correlation gives $\rho_{L P, z}=0.40$ with the p-value of $<10^{-10}$. Therefore, there is a positive correlation between disk luminosity and jet power in radio quasars.

The jet production efficiency is defined as

$$
\eta_{\mathrm{jet}} \equiv \frac{P_{\mathrm{jet}}}{\dot{M}_{\mathrm{in}} c^{2}}=\frac{P_{\mathrm{jet}}}{L_{\mathrm{disk}} / \varepsilon}
$$

The dashed line in Fig. $\square$ represents $P_{\text {jet }}=L_{\text {disk }} / \varepsilon=\dot{M}_{\text {in }} c^{2}$ with $\varepsilon=0.1$ corresponding to $\eta_{\text {jet }}=1$. The distribution of the jet production efficiency of our radio quasar samples are shown in Fig. $\square$. We show the cases of $\varepsilon=0.1$ and 0.3 , although the efficiency can be from 0.057 for a Schwarzschild $\mathrm{BH}$ to 0.42 for an extreme Kerr BH [25]. Here, $<\log \eta_{\text {jet }}>=-1.97 \pm 0.54$ corresponding to $\eta_{\mathrm{jet}} \simeq 1.1_{-0.76}^{+2.6} \times 10^{-2}$ for $\varepsilon=0.1$. Only 2 quasars have $\eta_{\mathrm{jet}} \geq 1$. This low jet production efficiency is consistent with the study by [5] in which they used a correlation between optical and radio luminosities of 763 FR-II quasars utilizing the SDSS-FIRST catalog. This low efficiency is not significantly enhanced even if we set $\varepsilon=0.3$ (D)). 


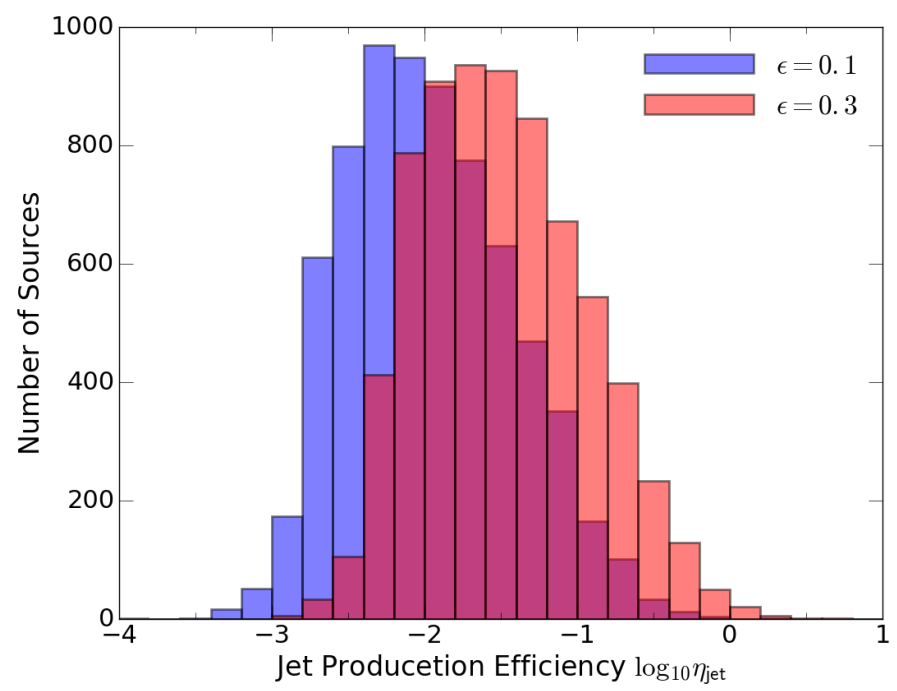

Figure 2: Distribution of the jet production efficiencies of our radio quasar samples. We assume the radiative efficiency of the disk $\varepsilon$ to be 0.1 (blue) and 0.3 (red).

\section{Fundamental Planes in Supermassive Black Holes}

The correlations between $M_{\mathrm{BH}}$ and $L_{\mathrm{disk}}$ or $P_{\text {jet }}$ are examined. For the correlation between $M_{\mathrm{BH}}$ and $L_{\mathrm{disk}}$, the Spearman rank correlation also gives $\rho_{M L, P}=0.37$ with the p-value of $<10^{-10}$. On the contrary, for the correlation between $M_{\mathrm{BH}}$ and $P_{\text {jet }}$, the Spearman rank correlation gives $\rho_{M P, L}=-0.028$ with the p-value of 0.019 . Thus, once we exclude the dependence on $L_{\mathrm{disk}}$, there is no correlation between $M_{\mathrm{BH}}$ and $P_{\mathrm{jet}}$ in radio-loud luminous quasars. Therefore, the disk luminosity correlates with both the $\mathrm{BH}$ mass and the jet power, while the jet power correlates with the disk luminosity, i.e. absolute mass accretion rate, but not with the $\mathrm{BH}$ mass. This result is consistent with semi-analytical studies of SMBH growth [B.5]].

\section{Discussions}

\subsection{Comparison with Blazar Studies}

The relation between disk and jet has been also investigated using blazar spectral fitting studies

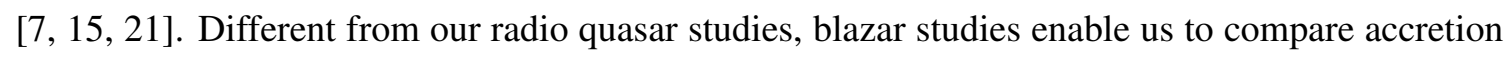
with jet outflow at the same epoch. Multi-wavelength observations from radio to gamma-ray allow us to study overall SED and physical parameters of jets via spectral fitting. A spectrum of a blazar is composed of two emission components: the low-energy component is synchrotron radiation and the other one is inverse Compton component targeting synchrotron radiation [23], [33] and/or external radiation field [Q, 5]].

Generally, blazar spectral fitting studies predict large jet production efficiencies $\eta_{\text {jet }} \sim 0.1-10$ [ [G, [15, [2]], while our results show $\eta_{\text {jet }} \simeq 1.1_{-0.76}^{+2.6} \times 10^{-2}$. Such a difference has also been recently reported by [40] using published blazar samples. Here, the jet power estimates based on blazar 
spectral fittings strongly rely on the assumptions on the pair fraction and the electron minimum Lorentz factor $\gamma_{e, \min }$ [2], 40]]. More pairs per one proton and/or higher $\gamma_{e \text {, min }}$ would reduce the jet power estimation based on blazar spectral fit. Although $\gamma_{\min }$ is known to be $\sim m_{p} / m_{e}$ for the terminal shocks of quasar jets [54], the minimum Lorentz factor of electrons in blazar jet is not well constrained. Recent NUSTAR observations revealed that electron spectrum extends down to electron energies of at least $\gamma_{e} \sim 10^{2}-10^{3}$ for nearby BL Lacs [24, [32]. If $\gamma_{\min }$ is determined by the mass ratio of a proton and an electron (i.e. $\gamma_{\min }=m_{p} / m_{e}$ ), the jet power can be an order of one to two lower than the case in which $\gamma_{\min }$ is determined by the energy ratio between protons and electrons [R]], which would lead a consistent result with our radio quasar studies.

\subsection{Implication on to the Jet Production}

Recent general relativistic magnetohydrodynamic (GRMHD) numerical studies showed that powerful relativistic jets are launched in the MAD which can confine magnetic flux on BHs by its ram pressure [57, [34]. Extracted jet power by the rotation of BHs threaded by magnetic fields, so-called the BZ power, is given by [四, [5], 56, 57]

$$
P_{\mathrm{BZ}}=4.0 \times 10^{-3} \frac{1}{c} \Omega_{\mathrm{H}}^{2} \Phi_{\mathrm{BH}}^{2} f\left(\Omega_{\mathrm{H}}\right) \simeq 10\left(\frac{\phi_{\mathrm{BH}}}{50}\right)^{2} x_{a}^{2} f\left(x_{a}\right) \dot{M}_{\mathrm{in}} c^{2},
$$

where $\Omega_{\mathrm{H}}=a c / 2 r_{\mathrm{H}}$ is the angular frequency of the $\mathrm{BH}$ horizon, $\Phi_{\mathrm{BH}}$ is the net magnetic field flux accumulated in the central region, $x_{a} \equiv r_{g} \Omega_{\mathrm{H}} / c$, and $f\left(x_{a}\right) \approx 1+1.38 x_{a}^{2}-9.2 x_{a}^{4} \cdot a \equiv J_{\mathrm{BH}} / J_{\mathrm{BH}, \max }=$ $c J_{\mathrm{BH}} / G M_{\mathrm{BH}}^{2}$ is the dimensionless $\mathrm{BH}$ spin parameter, $r_{\mathrm{H}}=r_{g}\left(1+\sqrt{1-a^{2}}\right)$ is the horizon radius, $r_{g}=G M_{\mathrm{BH}} / c^{2}$ is the gravitational radius of the $\mathrm{BH} . \phi_{\mathrm{BH}}=\Phi_{\mathrm{BH}} / \sqrt{\dot{M}_{\mathrm{in}} r_{g}^{2} c}$ is the dimensionless magnetic flux threading the $\mathrm{BH}$ and is typically on the order of 50 based on GRMHD simulations [B4]]. This gives $\eta_{\mathrm{jet}, \mathrm{BZ}} \equiv P_{\mathrm{BZ}} / \dot{M}_{\mathrm{in}} c^{2} \simeq 10\left(\phi_{\mathrm{BH}} / 50\right)^{2} x_{a}^{2} f\left(x_{a}\right)$.

Fig. 3$]$ gives the distribution of the dimennsionless spin parameter $a$ assuming $\varepsilon=0.1$ and $\phi_{\mathrm{BH}}=50$. For $\phi_{\mathrm{BH}}=50,<\log a>=-0.88 \pm 0.26$ corresponding to $a \simeq 0.13_{-0.059}^{+0.11}$. However, this spin parameter value is smaller than what is expected from the cosmological merger SMBH evolution models [60, 6], 62]. Fig. [3] also shows the spin parameter distribution but assuming $\phi_{\mathrm{BH}}=20$. In this case, we have $\langle\log a\rangle=-0.50 \pm 0.24$. Therefore, the results on the spin parameter strongly depend on the assumption on the magnetic flux threading the $\mathrm{BH}$ which is expected to be the dominant factor for the generation of jets considering the radio loundness distribution [50].

Observationally, various attempts have been recently considered to measure the magnetic field near active SMBHs. VLBI observations toward M 87, whose accretion rate is significantly subEddingtion [ए]], revealed $B \sim 10 \mathrm{G}$ at the jet base $\sim 10 R_{s}$ from the central BH using the synchrotron self-absorption (SSA) frequency [26, [Q] ]. Recent linear polarimetric adaptive optics observation of an AGN torus yields a dusty torus magnetic filed strength in the range of 4-82 mG assuming a clumpy torus model [R2]. Maser observations also constrains the magnetic fields at $\sim 0.2 \mathrm{pc}$ from the central $\mathrm{BH}$ to be $\lesssim 1 \mathrm{G}$ [ए6]. Although the B-field in the inner region (near the corona scale) has not been well investigated yet, detection of coronal synchrotron emission would provide an information on magnetic field [20, 42]. Very recently, [22] reported detection of coronal synchrotron emission from nearby seyferts which infer the magnetic field strength of $\sim 10 \mathrm{G}$ in the corona scale. 


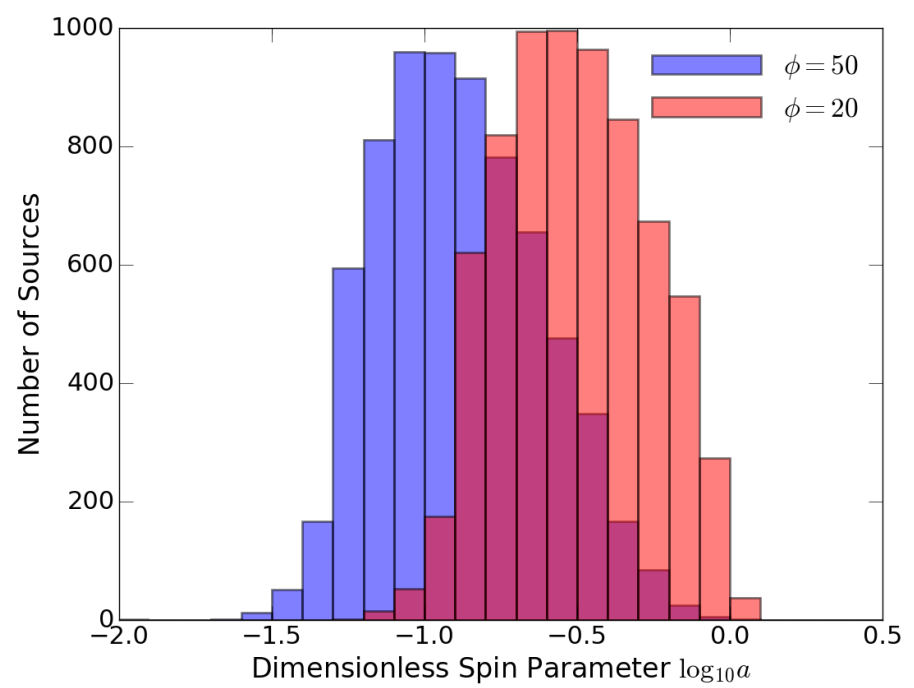

Figure 3: Distribution of the dimensionless spin parameters of our radio quasar samples. The dimensionless magnetic flux $\phi_{\mathrm{BH}}$ is set to be 50 (blue) and 20 (red). We assume the radiative efficiency of the disk $\varepsilon$ to be 0.1 .

\section{Conclusions}

In this paper, we studied the disk-jet connection in active SMBHs utilizing 7017 SDSS-NVSS detected radio-loud quasars up to $z=4.9$. We converted the $1.4 \mathrm{GHz}$ radio luminosity to the jet power using an empirical relation [63] which is calibrated by the X-ray cavity measurements [63, [7]]. Bolometric accretion disk luminosity is estimated by the SED templates in [45]. Central BH mass is also provided by [49] using a single-epoch spectrum.

We found that the quasar jet powers correlate with the bolometric disk luminosities. We have $\log P_{\text {jet }}=(0.96 \pm 0.012) \log L_{\text {disk }}+(0.79 \pm 0.55)$ with a scatter of 0.54 . The jet power rarely exceeds the accretion luminosity. By assuming the accretion disk efficiency of $\varepsilon=0.1$, we further found that the jet production efficiency is $\eta_{\text {jet }} \simeq 1.1_{-0.76}^{+2.6} \times 10^{-2}$. These results do not significantly change even if we adopt various different selection criteria or the higher disk efficiency of $\varepsilon=0.3$. $\eta_{\text {jet }}$ gradually increases with the limiting radio loudness, although it will be still at an order of 0.1 even for $R_{\lim } \gtrsim 10^{3}$.

We further tested the existence of the fundamental plane among $M_{\mathrm{BH}}, L_{\mathrm{disk}}$, and $P_{\text {jet }}$ for quasars at $0.01 \lesssim \lambda$. We could not find a statistically significant correlation between $M_{\mathrm{BH}}$ and $P_{\text {jet }}$ excluding the dependence on $L_{\text {disk. }}$ This implies that the plane would not exist for radio-loud quasars in the standard accretion regime. This is consistent with recent studies which revealed that the plane exists only for low accretion AGNs [35], 4⿴囗十]].

Following the BZ scenario [䧃], the jet power is related to the $\mathrm{BH}$ spin and the magnetic flux threading the BH. Taking the value of the dimensionless magnetic flux value from numerical simulations by [B4] $\phi_{\mathrm{BH}}=50$, we estimated the distribution of the dimensionless spin parameters $a$. However, the resulting $a$ is $\simeq 0.13_{-0.059}^{+0.11}$ which is much smaller than what is expected from the 
cosmological merger SMBH evolution [62]. Therefore, the magnetic flux strength threading the SMBHs might be weaker than it is expected from numerical simulations following the spin evolutionary models. Magnetic field measurements in the vicinity of the central BHs would help us to understand the detailed BZ processes. The resulting low jet efficiency can be also due to dependence of the efficiency on geometrical thickness of the accretion flow [四], since our quasar samples accreting at $0.01 \lesssim \lambda_{\text {disk }} \lesssim 0.3$ is expected to be have small geometrical thickness.

\section{References}

[1] Avara, M. J., McKinney, J. C., \& Reynolds, C. S. 2016, MNRAS , 462, 636

[2] Baum, S. A., \& Heckman, T. 1989, ApJ , 336, 702

[3] Becker, R. H., White, R. L., \& Helfand, D. J. 1995, ApJ , 450, 559

[4] Blandford, R. D., \& Znajek, R. L. 1977, MNRAS , 179, 433

[5] Blundell, K. M., \& Rawlings, S. 2000, AJ , 119, 1111

[6] Buttiglione, S., Capetti, A., Celotti, A., et al. 2010, A\&A , 509, A6

[7] Celotti, A., \& Ghisellini, G. 2008, MNRAS , 385, 283

[8] Condon, J. J., Cotton, W. D., Greisen, E. W., et al. 1998, AJ , 115, 1693

[9] Dermer, C. D., \& Schlickeiser, R. 1993, ApJ , 416, 458

[10] Di Matteo, T., Allen, S. W., Fabian, A. C., Wilson, A. S., \& Young, A. J. 2003, ApJ , 582, 133

[11] Elvis, M., Wilkes, B. J., McDowell, J. C., et al. 1994, ApJS , 95, 1

[12] Fabian, A. C. 2012, ARA\&A, 50, 455

[13] Fanaroff, B. L., \& Riley, J. M. 1974, MNRAS , 167, 31P

[14] Fukugita, M., Ichikawa, T., Gunn, J. E., et al. 1996, AJ , 111, 1748

[15] Ghisellini, G., Tavecchio, F., Maraschi, L., Celotti, A., \& Sbarrato, T. 2014, Nature , 515, 376

[16] Gnedin, Y. N., Globina, V. N., Piotrovich, M. Y., Buliga, S. D., \& Natsvlishvili, T. M. 2014, Astrophysics, 57, 163

[17] Godfrey, L. E. H., \& Shabala, S. S. 2013, ApJ , 767, 12

[18] Gunn, J. E., Siegmund, W. A., Mannery, E. J., et al. 2006, AJ , 131, 2332

[19] Hada, K., Kino, M., Doi, A., et al. 2016, ApJ , 817, 131

[20] Inoue, Y., \& Doi, A. 2014, PASJ , 66, L8

[21] Inoue, Y., \& Tanaka, Y. T. 2016, ApJ , 828, 13

[22] Inoue, Y., \& Doi, A. 2018, ApJ , 869, 114

[23] Jones, T. W., O’dell, S. L., \& Stein, W. A. 1974, ApJ , 188, 353

[24] Kataoka, J., \& Stawarz, Ł. 2016, ApJ , 827, 55

[25] Kato, S., Fukue, J., \& Mineshige, S., eds. 1998, Black-hole accretion disks

[26] Kino, M., Takahara, F., Hada, K., et al. 2015, ApJ , 803, 30 
[27] Komissarov, S. S., Barkov, M. V., Vlahakis, N., \& Königl, A. 2007, MNRAS , 380, 51

[28] Kozieł-Wierzbowska, D., \& Stasińska, G. 2011, MNRAS , 415, 1013

[29] Lopez-Rodriguez, E., Packham, C., Jones, T. J., et al. 2015, MNRAS , 452, 1902

[30] Lu, Y., Wang, T., Zhou, H., \& Wu, J. 2007, AJ , 133, 1615

[31] Maccarone, T. J., Gallo, E., \& Fender, R. 2003, MNRAS , 345, L19

[32] Madejski, G. M., Nalewajko, K., Madsen, K. K., et al. 2016, ArXiv e-prints, arXiv:1609.02203

[33] Maraschi, L., Ghisellini, G., \& Celotti, A. 1992, ApJL , 397, L5

[34] McKinney, J. C., Tchekhovskoy, A., \& Blandford, R. D. 2012, MNRAS , 423, 3083

[35] Merloni, A., \& Heinz, S. 2008, MNRAS , 388, 1011

[36] Merloni, A., Heinz, S., \& di Matteo, T. 2003, MNRAS , 345, 1057

[37] Mullin, L. M., Riley, J. M., \& Hardcastle, M. J. 2008, MNRAS , 390, 595

[38] O’Sullivan, E., Giacintucci, S., David, L. P., et al. 2011, ApJ , 735, 11

[39] Pâris, I., Petitjean, P., Aubourg, É., et al. 2014, A\&A , 563, A54

[40] Pjanka, P., Zdziarski, A. A., \& Sikora, M. 2016, ArXiv e-prints, arXiv:1607.08895

[41] Plotkin, R. M., Markoff, S., Kelly, B. C., Körding, E., \& Anderson, S. F. 2012, MNRAS , 419, 267

[42] Raginski, I., \& Laor, A. 2016, MNRAS , 459, 2082

[43] Rawlings, S., \& Saunders, R. 1991, Nature , 349, 138

[44] Rawlings, S., Saunders, R., Eales, S. A., \& Mackay, C. D. 1989, MNRAS , 240, 701

[45] Richards, G. T., Lacy, M., Storrie-Lombardi, L. J., et al. 2006, ApJS , 166, 470

[46] Saunders, R., Baldwin, J. E., Rawlings, S., Warner, P. J., \& Miller, L. 1989, MNRAS , 238, 777

[47] Schneider, D. P., Richards, G. T., Hall, P. B., et al. 2010, AJ , 139, 2360

[48] Shabala, S. S., \& Godfrey, L. E. H. 2013, ApJ , 769, 129

[49] Shen, Y., Richards, G. T., Strauss, M. A., et al. 2011, ApJS , 194, 45

[50] Sikora, M., \& Begelman, M. C. 2013, ApJL, 764, L24

[51] Sikora, M., Begelman, M. C., \& Rees, M. J. 1994, ApJ , 421, 153

[52] Sikora, M., Stasińska, G., Kozieł-Wierzbowska, D., Madejski, G. M., \& Asari, N. V. 2013, ApJ , 765, 62

[53] Sikora, M., Stawarz, Ł., \& Lasota, J.-P. 2007, ApJ , 658, 815

[54] Stawarz, Ł., Cheung, C. C., Harris, D. E., \& Ostrowski, M. 2007, ApJ , 662, 213

[55] Tanabe, K., \& Nagataki, S. 2008, Phys. Rev. D, 78, 024004

[56] Tchekhovskoy, A., Narayan, R., \& McKinney, J. C. 2010, ApJ , 711, 50

[57] —. 2011, MNRAS , 418, L79

[58] Terashima, Y., \& Wilson, A. S. 2003, ApJ , 583, 145

[59] van Velzen, S., \& Falcke, H. 2013, A\&A , 557, L7 
[60] Volonteri, M., Madau, P., Quataert, E., \& Rees, M. J. 2005, ApJ , 620, 69

[61] Volonteri, M., Sikora, M., \& Lasota, J.-P. 2007, ApJ , 667, 704

[62] Volonteri, M., Sikora, M., Lasota, J.-P., \& Merloni, A. 2013, ApJ , 775, 94

[63] Willott, C. J., Rawlings, S., Blundell, K. M., \& Lacy, M. 1999, MNRAS , 309, 1017

[64] Woo, J.-H., \& Urry, C. M. 2002, ApJ , 579, 530

[65] York, D. G., Adelman, J., Anderson, Jr., J. E., et al. 2000, AJ , 120, 1579

[66] Zakamska, N. L., \& Greene, J. E. 2014, MNRAS , 442, 784

[67] Zirbel, E. L., \& Baum, S. A. 1995, ApJ , 448, 521 